\title{
Strategies for Complete Proteomic Analysis of Hydrophobic Proteins in Complex Biological Samples - Hyde-and Seek
}

\section{Djuro Josic*}

Analytical Biochemistry and Proteomics, Department of Biotechnology, University of Rijeka, Croatia

The completeness of the proteomic analysis of complex biological samples frequently depends on two important factors, proper sample preparation and thorough data analysis. However, the first one is frequently neglected. The early strategies for such analyses of cells or tissues and organs started with the protein extraction out of crude homogenates or body fluids followed by proteolytic (most frequently tryptic) digestion or chemical cleavage, followed by (1 or 2D) chromatographic separation and mass spectrometric analysis of resulting peptides (LC-MS, or LC-MS/MS). Alternatively, the sample was separated by use of 1 - or 2-D electrophoresis followed by excision of polypeptide bands or spots and tryptic digestion and again by LC-MS/MS analysis [1]. However, it turned out that the performed extraction step was not complete, and a significant part of proteins in the sample were lost. Additionally, without targeted fractionation, the highly abundant as well as more hydrophilic proteins in complex samples often masked the less abundant proteins and proteins that contain hydrophobic domains and posttranslational modifications (PTMs). It frequently results in limited detection of these proteins by mass spectrometry $[2,3]$.

The targeted fractionation includes the isolation subfractions that contain either organelles or macromolecular structures of the cell and/or biological fluids. Separation of complex startng materials into multiprotein fractions substantially increases the probability of detecting low abundance proteins, and terms such as "subcellular proteomics" or "organelle proteomics" have been established $[3,4]$. Methods for subcellular fractionation are e.g. differential centrifugation, electrophoresis and affinity fractionation. The next step is the destruction of the sample structures, mostly by use of different solubilization techniques followed by chromatographic and/ or electrophoretic separation, mostly according to size, hydrophobicity or charge of macromolecular components of these complex biological mixtures. Hydrophobic proteins, especially proteins located in plasma membranes and other cellular membranes have a special position in proteome research due to their physiological roles, diversity, and behavior during the process of purification and separation. Additionally, most of them have also several posttranslational modifications (PTMs). These modifications are frequently the reason for their extremely high microheterogeneity. Both protein hydrophobicity that is caused by the presence of one of more differently long hydrophobic domains, and their high degree of glycosylation and/or phosphorylation causes difficulties in the separation process such as 1 and 2-D electrophoresis and liquid chromatography [3].

Because of the hydrophobicity and microheterogeneity of such proteins that is caused by both presence of hydrophobic sequences and PTMs, it has become necessary to develop new strategies for their proteomic analysis [3,5]. An early developed and frequently very efficient strategy is the selective solubilization of proteins out of their sub-cellular structures according to their hydrophobicity. This method was developed more than 30 years ago [6] and has been applied with gel-based or gel-free methodologies as a sample preparation technique e.g. for proteomic analyses of hydrophobic, integral membrane proteins $[3,5,7]$.
The limited or sometimes total insolubility of hydrophobic proteins such as integral membrane proteins in pure aqueous media interferes with their proteolytic digestion. It is a well-known and frequently discussed topic $[3,5,7]$. The frequent consequence in proteomic analysis is their under-representation in the lists of identified proteins. It is due the fact that protein identification and quantification by use of tandem mass spectrometry (MS/MS) is highly dependent upon the generation of many unique tryptic (or proteolytic) fragments that can be separated by liquid chromatography. Most frequently used separation methods for peptide fractionation in the front of MS/MS analysis are reversed phase chromatography (RPC), or cation-exchange chromatography followed by RPC. Low abundance of hydrophobic proteins in complex biological samples, possible presence of PTMs, and the lack of charged residues, e.g. Arg/Lys in the case of tryptic digestion in hydrophobic domains are additional reasons why the hydrophobic proteins are frequently underrepresented in large-scale proteomic investigations [2,8]. Limited water solubility is also the main reason for under-representation of hydrophobic proteins after sample preparation by use of 1- and 2-D gel electrophoresis [9]. Together with the necessity to shorten the analysis time, the loss of hydrophobic proteins during sample preparation by use of electrophoretic separation was one of the main reasons to develop an alternative to gel-based proteomics for analyses of hydrophobic proteins. One of first methods is the multidimensional LC-MS strategy exemplified by solubilization and tryptic digestion of highly hydrophobic proteins in a buffer containing $60 \%(\mathrm{v} / \mathrm{v})$ methanol [10]. This protocol for sample preparation represents an important step toward improved detection of hydrophobic proteins by mass spectrometry.

The so-called "tube gel digestion" is an optimized method for enchanced proteolytic cleavage and subsequent detection of very hydrophobic proteins. Very big advantage of this method is that proteins can be previously solubilized by different detergents and/ or other reagents such as urea and guanidinium hydrochloride [11]. The solubilization agents can be subsequently washed out while the proteins are still immobilized in the gel matrix and can be subsequently digested. Using this approach, a major improvement in the detection of very hydrophobic peptides from water insoluble proteins in the subsequent mass spectrometric analysis could be achieved [12]. An optimized sample preparation method by use of stepwise solubilization

${ }^{*}$ Corresponding author: Prof. Djuro Josic, Analytical Biochemistry and Proteomics, Department of Biotechnology, University of Rijeka, Croatia, Tel: 140144-44427; E-mail: djuro_josic@brown.edu

Received January 22, 2014; Accepted January 24, 2014; Published January 26 2014

Citation: Josic D (2014) Strategies for Complete Proteomic Analysis of Hydrophobic Proteins in Complex Biological Samples - Hyde-and Seek. J Data Mining Genomics Proteomics 5: e111. doi:10.4172/2153-0602.1000e111

Copyright: (c) 2014 Josic D. This is an open-access article distributed under the terms of the Creative Commons Attribution License, which permits unrestricted use, distribution, and reproduction in any medium, provided the original author and source are credited. 
Citation: Josic D (2014) Strategies for Complete Proteomic Analysis of Hydrophobic Proteins in Complex Biological Samples - Hyde-and Seek. J Data Mining Genomics Proteomics 5: e111. doi:10.4172/2153-0602.1000e111

Page 2 of 2

with different salt solutions followed by detergent treatment and treatment with chaotropic reagents also results in protein fractionation according to their hydrophobicity. Additional pre-digestion with a protease previous to the modified "tube gel digestion" approach is an additional way to improve detection of very hydrophobic peptides in mass spectrometry. During this pre-treatment the loss of some less hydrophobic proteins can occur, but it enables the detection of additional very hydrophobic ones, e.g. containing more than 4 transmembrane domains (TMDs) [12]. Contrary to other experiences [13], an additional pre-treatment with a glycosidase such as PGNase F did not yield detection of additional hydrophobic proteins.

The introduction of a mass spectrometer such as Orbitrap with high sensitivity, high mass resolving power and mass accuracy and faster scan rate is the further way to significantly improve detection of low abundance proteins, especially the hydrophobic ones [12]. It can be demonstrated with the detection of the solute carrier organic anion transporter family member $1 \mathrm{~B} 2$ in a plasma membrane fraction of rat liver solubilized with the non-ionic detergent Triton X100. This protein contains 12 TMDs, and could not be detected if a less sensitive mass spectrometer was applied for the analysis of the same sample [14], but it could be detected with sequence coverage of $4.8 \%$ after introduction of the high sensitive Orbitrap mass spectrometer. The sample preparation by use of selective extraction resulted in a improved sequence coverage $(11.1 \%)$ that was additionally increased after tryptic pre-digestion (14.7\%). This effect was even higher for integrin $B 1$ in the same sample. It is an integral membrane protein with 1 TMD. The selective solubilization did not contribute to better coverage (about $4.8 \%$ for both, control and selectively solubilized sample). However, the sequence coverage increased to 12.4 after tryptic pre-digestion [12]. These and similar experiences demonstrate the necessity to use special strategies for detection of hydrophobic proteins. The best way seems to be a combination of different methods for sample preparation and a protocol for proteolytic digestion that enables better accessibility of hydrophobic region for enzymatic (or chemical) cleavage. The use of a sensitive up-to date mass spectrometer is essential, and corresponding software also enables quantitative determination of proteins in biological samples. It is an important step on the way for identification and validation e.g. of potential disease biomarker candidates [12].

\section{References}

1. Issaq HJ (2001) The role of separation science in proteomics research Electrophoresis 22: 3629-3638.

2. Eichacker LA, Granvogl B, Mirus O, Müller BC, Miess C, et al. (2004) Hiding behind hydrophobicity. Transmembrane segments in mass spectrometry. J Biol Chem 279: 50915-50922.

3. Josic Dj, Clifton JG (2007) Mammalian plasma membrane proteomics. Proteomics 7: 2874-2885.

4. Huber LA, Pfaller K, Vietor I (2003) Organelle proteomics: implications for subcellular fractionation in proteomics. Circ Res 92: 962-968.

5. Cordwell SJ, Thingholm TE (2010) Technologies for plasma membrane proteomics. Proteomics 10: 611-627.

6. Fujiki Y, Hubbard AL, Fowler S, Lazarow PB (1982) Isolation of intracellular membranes by means of sodium carbonate treatment: application to endoplasmic reticulum. J Cell Biol 93: 97-102.

7. Speers AE, Wu CC (2007) Proteomics of integral membrane proteins--theory and application. Chem Rev 107: 3687-3714

8. Helbig AO, Heck AJ, Slijper M (2010) Exploring the membrane proteome-challenges and analytical strategies. J Proteomics 73: 868-878.

9. Rabilloud T (2009) Membrane proteins and proteomics: love is possible, but so difficult. Electrophoresis 30 Suppl 1: S174-180.

10. Blonder J, Chan KC, Issaq HJ, Veenstra TD (2006) Identification of membrane proteins from mammalian cell/tissue using methanol-facilitated solubilization and tryptic digestion coupled with 2D-LC-MS/MS. Nat Protoc 1: 2784-2790.

11. Lu X, Zhu H (2005) Tube-gel digestion: a novel proteomic approach for high throughput analysis of membrane proteins. Mol Cell Proteomics 4: 1948-1958.

12. Cao L, Clifton JG, Reutter W, Josic D (2013) Mass spectrometry-based analysis of rat liver and hepatocellular carcinoma Morris hepatoma 7777 plasma membrane proteome. Anal Chem 85: 8112-8120.

13. Wollscheid B, Bausch-Fluck D, Henderson C, O’Brien R, Bibel M, et al. (2009) Mass-spectrometric identification and relative quantification of $\mathrm{N}$-linked cel surface glycoproteins. Nat Biotechnol 27: 378-386.

14. Clifton JG, Li X, Reutter W, Hixson DC, Josic D (2007) Comparative proteomics of rat liver and Morris hepatoma 7777 plasma membranes. J Chromatogr B Analyt Technol Biomed Life Sci 849: 293-301. 